\title{
Chapter 16 \\ Plastic Lab-on-Chip for the Optical Manipulation of Single Cells
}

\author{
Rebeca Martínez Vázquez, Gianluca Trotta, Annalisa Volpe, \\ Melania Paturzo, Francesco Modica, Vittorio Bianco, Sara Coppola, \\ Antonio Ancona, Pietro Ferraro, Irene Fassi and Roberto Osellame
}

\begin{abstract}
Lab-on-chips (LoCs) are microsystems capable of manipulating small amounts of fluids in microfluidic channels. They have a huge application potential, from basic science to chemical synthesis and point-of-care medical analysis. Polymers are rapidly emerging as the substrate of choice for LoC production, thanks to a low material cost and ease of processing. Two breakthroughs that could promote LoC diffusion are a microfabrication technology with cost-effective and rapid prototyping capabilities and also an integrated on-chip optical detection system. This chapter proposes the use of femtosecond laser micromachining combined with microinjection moulding as a novel highly-flexible microfabrication platform for polymeric LoCs with integrated optical detection, for the realization of low-cost and truly portable biophotonic microsystems. We demonstrate a LoC for the relevant application of non-invasive and contactless mechanical phenotyping of single cancer cells.
\end{abstract}

R. M. Vázquez · R. Osellame ( $₫)$

CNR-IFN, Istituto di Fotonica e Nanotecnologie, Milan, Italy

e-mail: roberto.osellame@polimi.it

G. Trotta $\cdot$ F. Modica

CNR-STIIMA, Istituto di Sistemi e Tecnologie Industriali Intelligenti per il Manifatturiero Avanzato, Bari, Italy

A. Volpe $\cdot$ A. Ancona

CNR-IFN, Istituto di Fotonica e Nanotecnologie, Bari, Italy

M. Paturzo $\cdot$ V. Bianco $\cdot$ S. Coppola $\cdot$ P. Ferraro

CNR-ISASI, Istituto di Scienze Applicate e Sistemi Intelligenti "Eduardo Caianiello",

Pozzuoli, NA, Italy

I. Fassi

CNR-STIIMA, Istituto di Sistemi e Tecnologie Industriali Intelligenti per il Manifatturiero

Avanzato, Milan, Italy

(C) The Author(s) 2019

T. Tolio et al. (eds.), Factories of the Future,

https://doi.org/10.1007/978-3-319-94358-9_16 


\subsection{Scientific and Industrial Motivations}

Point-of-care analysis is one of the great challenges in many application fields, from healthcare to environmental monitoring [1]. The widespread diffusion of electronic portable devices enables data analysis in-field; however, the bottleneck is often represented by the miniaturization of the sensor. While electric sensors, e.g. accelerometers, are effectively integrated already on cell-phones and watches, microfluidic devices still suffer from a limited diffusion, albeit they could represent the most interesting devices for personalized healthcare, since they give access to biological fluid monitoring. Such miniaturized microfluidic devices are often called Lab-onChip (LoC) because they aim at integrating in a small chip a whole set of analysis on a given fluid that would normally require an entire laboratory $[2,3]$. Among the advantages of a LoC approach, it is worth highlighting the point-of-care use, the limited amount of liquid sample required, the full automation of the analysis process that can be performed by non-specialized personnel. Notwithstanding their huge application potential, LoC penetration in the market is limited by the development cost and by the difficulty in integrating the analysis apparatus on the same chip where the fluid manipulation is performed. Two improvements that could promote LoC diffusion are:

(i) A microfabrication technology with low-cost and rapid prototyping capabilities.

(ii) On-chip optical components for detection and/or manipulation of the fluidic sample.

These two features will significantly decrease the cost for LoC development and greatly enhance the compactness and portability of the devices, respectively. In the following we will introduce and outline the main ingredients that one may want to combine into a new generation of LoC devices.

Most of the commercial LoCs are made in polymeric materials due to their low cost and easy processing [4]. Amorphous polymers are completely transparent to wavelengths in the UV-visible range, and their optical properties can be easily tuned by changing the composition. Many of them are already commercially available products, but an almost unlimited number of modifications in the composition are feasible for a further optimization of the chosen structure.

The most widely employed manufacturing technology is microinjection moulding since it enables large volume and high precision production of plastic devices. This technology requires the fabrication of moulds and tools that are quite expensive and limit the reconfigurability of the LoC layout.

A completely different fabrication approach is based on laser micromachining as a flexible and rapid prototyping process for the creation of a variety of microstructures in a broad range of materials. In particular, femtosecond lasers, due to their peculiar light-matter interaction regime, offer unique microfabrication capabilities both with transparent (e.g. polymeric) and non-transparent (e.g. metallic) materials [5]. Recent technological advances have dramatically improved the performance and reliability of femtosecond laser systems, making them available as a practical micromachining 
tool $[6,7]$. However, this technology is mainly used only in academic laboratories because it is too expensive for large batch production.

The analysis of biological fluids often requires optical means, from microscope imaging to fluorescence detection. These operations are currently performed with external and bulky equipment, thus frustrating the miniaturization of the fluidic handling of the sample [8]. Digital Holographic Microscopy (DHM) is a powerful method to visualize biological cells [9]. Not only it provides an enhanced view of transparent objects but it also allows their three-dimensional reconstruction. In addition, it enables software-based autofocus and aberration correction thus greatly simplifying the optical imaging system; this makes DHM a very suitable approach for an integrated, highly-informative and low-cost microscopy set-up.

Herein, we propose the combination of the above elements to produce a highly flexible microfabrication platform for polymeric LoCs with integrated optical imaging, for the realization of low-cost and truly portable biophotonic microsystems.

The unique integration of photonics and microfluidics, offered by the assembly of optical components in the biochips, will enable to implement a wealth of novel functionalities. In this work, we will focus on a specific polymeric device that has relevant application potentials and that demonstrates the main features of our new approach. We will present a LoC for mechanical phenotyping of single cells exploiting optical forces on the chip.

The chapter is organized as follows. In Sect. 16.2 we will discuss the state of the art of all the techniques that we will exploit in this work. In Sect. 16.3 we will detail the basic concepts behind the innovative approach we are proposing, and we will discuss how it will allow to overcome the current limitations in the LoC manufacturing industry. In Sect. 16.4 we will introduce in details the technologies, methods and tools of our new approach. In Sect. 16.5 we will present the results that we achieved and, in particular, we will present the LoC device for single-cell mechanical phenotyping as an example of a complex analysis that can be performed in a simple device efficiently manufactured. Finally, in Sect. 16.6 we will draw some conclusions and discuss the perspectives of our work.

\subsection{State of the Art}

The increasing interest in microfluidic devices for healthcare and diagnostic applications, such as Lab on Chips (LoCs), pushed the research towards the evaluation of alternative manufacturing technologies capable of reducing the production costs and guaranteeing a mass production of ready-to-use micro structured devices. Among different micro-moulding techniques, micro-injection moulding ( $\mu \mathrm{IM})$ is a promising process for manufacturing these polymeric disposable microfluidic devices [10].

$\mu \mathrm{IM}$ is a process capable of reproducing the micron or submicron features of metallic moulds to a polymeric product. The granular material is transferred from a hopper into a plasticizing unit to be made molten and soft. Then the material is forced inside a mould cavity where a holding pressure is applied for a specific time 
to compensate the shrinking of the material. After enough time, the material freezes into the mould shape, then it is ejected to repeat the cycle.

Polymers are a wide family of materials characterized by a large spectrum of properties, thus it is relatively easy to find the proper material that has the required properties for processing and application. A typical polymer can completely fill and accurately replicate small features down to tens of nanometres, when the optimum processing conditions are applied [11]. Among all polymers, Poly(methyl methacrylate) (PMMA) is appropriate for lab-on-chip applications because it is mechanically strong, optically transparent, biocompatible and it has good chemical stability. Microinjection moulding of PMMA is a well-established process and it is possible to obtain LoCs with extremely small features [12-14], if the process is combined with UV lithography coupled with electrodeposition (LIGA process) for the manufacture of nickel mould inserts.

Femtosecond laser micromilling is an ablation procedure causing the vaporization of the material in a layer-by-layer fashion because of the interaction between the laser beam and the work piece that is machined. This technology is becoming important for applications in rapid prototyping and manufacturing of miniaturized metallic, semiconductor as well as ceramic and glass components or devices. Femtosecond pulses provide high precision and reduced thermal damages and/or structural modifications with respect to longer laser pulses [15, 38].

Besides pulse duration, several parameters have a direct impact on the accuracy of the laser milling process and the quality of the microstructured surfaces, i.e. the pulse repetition rate and laser fluence. Even in the ultrashort pulse regime, it is always advisable to work with near threshold fluence because for higher average powers the ablation mechanism is once again dominated by melting [15]. Furthermore, several studies have clearly shown that the process strategy significantly affects the resulting surface quality and edge sharpness $[16,17]$. In particular, the surface roughness and its homogeneity are highly influenced by the beam-scanning pattern.

Femtosecond laser ablation offers several advantages for the fabrication of microfluidic chip on polymer substrates. Besides its geometrical flexibility and 3D capabilities, the ultrashort deposition of energy in the material causes vaporization before thermal diffusion can occur, thus minimizing the heat-affected zone and increasing the accuracy of the ablated structures (if compared with longer pulses or continuous wave laser micromachining) [18]. This technology is particularly useful for the direct and rapid prototyping of devices, since it does not need the use of moulds or masks. Thus, the LoC is created by laser ablation along the pattern of the desired microfluidic scheme.

Besides material removal, femtosecond lasers are also used for welding different transparent materials at their interface by exploiting localized melting and resolidification below the ablation threshold. Several studies have demonstrated the ability of focused femtosecond laser pulses to weld fused silica [19], borosilicate and/or dissimilar glasses [20]. Tamaki et al. [21] achieved a first demonstration of fs-laser welding of silica glass in the low repetition rate regime $(1-200 \mathrm{kHz})$. In their study, the laser focus was accurately placed at the interface of two glass specimens. The focal region was elongated along the optical axis and across the two material interface 
due to filamentation generated by the balance between self-focusing and diffraction [22]. The intensity in the filamentary region was high enough to induce localized melting and subsequent resolidification and joining.

Another reported approach to weld fused silica exploits ultrashort laser pulses at repetition rates in the $\mathrm{MHz}$ regime. In this case no filamentation occurs and the energy accumulation mechanism between subsequent pulses is at the basis of the material modification. High temperature hotspots close to the interface to be welded are produced, causing once again material melting and resolidification over volumes larger than the focal one. Even though the laser is focused on one material, heat diffusion causes melting and resolidification of both materials across the interface. Femtosecond laser welding of glass substrates in this regime has been reported by several works $[19,23]$. Welding with high repetition rate lasers has several advantages compared to the low repetition rate regime because a much higher processing speed is accessible and the size of the structural modification can be easily tuned by adjusting the writing speed.

The capability to observe the samples inside LoCs is crucial for monitoring and extracting information from the processes occurring inside the microfluidic channels. To this aim, the standard imaging techniques have been adjusted to be applied for the investigation of biological specimens in LoCs, novel diagnostic tools specifically tailored for LoCs have been proposed and new strategies to support the integration of the diagnostic and imaging functionalities on chip have been investigated. The novel imaging strategies to study biological samples in microfluidic channels need to satisfy the following requirements:

- quantitative information is needed to better understand the cell structure and behaviour;

- compact portable devices are needed to use LoCs for point-of-care diagnostics;

- a high-throughput imaging system needs to be developed to obtain statistically relevant information by analysing multiple specimens in a reduced time period;

- label-free methods are favourite to elude sample pre-treatment and undesired alterations of the specimens.

Up to now, the two main advanced techniques for on-chip microscopy are the Digital in Line Holography (DILH) and the Holographic Opto-fluidic Microscopy (HOM), both based on a lensless approach. The use of DILH technique as imaging tool for LoC devices is quite limited because of the cost and size of coherent sources [24].

In this work we aim at demonstrating a final device for mechanical phenotyping of single cells. This requires the capability of characterizing the viscoelastic response of single cells to an external mechanical stimulus. In fact, cell deformability is an important aspect in biological analysis of cell functionalities and health state, resulting in a sensitive and label-free marker to monitor physiological or pathological changes in the cell. The study of cell mechanics can be a powerful tool to predict complex cell changes in a fast and more comprehensive way than the single analysis of biochemical or morphological parameters. This approach has already been validated for the detection of malignant change in cells [25]. Mechanical analysis of 
single cells is performed by exploiting optical forces in a double counterpropagating optical trap [26, 27]. Two optical waveguides or fibres, perfectly aligned on the two sides of a microfluidic channel, can trap a transparent particle through the optical forces exerted by the light emitted from the two sides. Increasing the optical power in the trap it is possible to also stretch the cell. By continuously imaging the cell contour one can retrieve the cell deformation and thus characterize the deformability cell by cell. Optical cell trapping and stretching has been predominantly a scientific tool, with no commercial or biomedical impact yet. However, cancer diagnosis with the optical stretcher has the potential for a broad impact on public health, since it can analyse very small number of cells with high sensitivity in a fast and reliable way, thus enabling early detection of a disease.

\subsection{Problem Statement and New Approach}

The new approach that we present in this work aims at satisfying the following requirements:

- use of polymers as materials for LoCs;

- development of a rapid prototyping technique;

- need for a low-cost fabrication technique for mass production;

- need for integrated imaging system for on-chip analysis.

Although $\mu \mathrm{IM}$ has demonstrated to be an excellent technique for mass production of polymer devices, it shows its weaknesses in a still non-mature market, where prototyping of new devices and small batch productions is the most common requirement. The limit of $\mu$ IM mainly resides in the low reconfigurability of the mould itself and in the high precision required to reproduce the micron-sized features. The common effects of polymer creep or shrinkage in the final products imposes an accurate design optimization of both the device and the mould. In addition, improved mould manufacturing strategies and control of the moulding conditions are required to allow $\mu \mathrm{IM}$ to become a reliable mass production technology that helps the spreading of microfluidic devices.

In this work, we present a variation of the traditional $\mu$ IM process that makes it a versatile micro-manufacturing platform for the production of LoCs. In fact, we will cope with the weak points of $\mu$ IM thanks to a synergic combination with femtosecond-laser-micromachining, thus creating a manufacturing process that is both effective for the prototyping and for the mass production.

Femtosecond laser micromachining will be employed in different steps of the LoC production cycle in order to provide the following advantages: (i) rapid prototyping of LoCs by direct laser ablation of a polymeric substrate; (ii) tool fabrication by laser milling of steel for mass production of the LoC by microinjection moulding; (iii) direct welding of polymeric slides for sealing the microfluidic network of the LoC without the addition of any glue. 
The possibility to use femtosecond lasers for rapid and precise structuring of steel is particularly effective when associated to a novel approach to microinjection moulding, based on the use of application-tailored removable inserts in a standardized mould. This approach will greatly enhance the versatility of $\mu$ IM. In fact, this approach will require the substitution of only small parts in the mould (the inserts) when changing the geometrical features of the device. The small steel inserts can be effectively micromachined by high-resolution femtosecond laser ablation without the drawback of long processing time that would be required to machine the whole mould.

A further critical point in the fabrication of plastic microfluidic devices is the sealing of the channels. Currently, the most widely used method for joining polymer parts is adhesive or thermal pressure bonding. The critical aspects of these approaches are the blocking of the channel by adhesive overflow and the damage of the microstructures due to the high temperatures and pressures that are applied. For these reasons, we have developed a novel fs-laser-based welding technique that can be exploited to join the fabricated PMMA slabs to obtain the final, closed, LoC device [28].

As previously discussed, holographic methods have been demonstrated to be quite successful for the imaging of specimens flowing inside microfluidic chips. However, the traditional lensless approach represents an inner limitation to the available resolution. Proper algorithms, mainly based on subpixel shifting, are needed to overcome the limit imposed by the pixel size [29]. As an alternative, we propose a lens-based approach that integrates the imaging functions on the chip by fabricating the lenses directly on top of it. We will use a method based on a pyro-electrohydrodynamic ink-jet technique that allows to deposit polymer drops on a polymer substrate [30].

\subsection{Developed Technologies, Methodologies and Tools}

In this section we will provide a technical discussion of the technologies and methodologies that we exploit in our new approach for LoC manufacturing. We will discuss the removable inserts concept (Sect. 16.4.1) and their micromachining with femtosecond lasers (Sect. 16.4.2.2). We will also discuss the use of femtosecond lasers to seal the LoCs (Sect. 16.4.3) and the use of forward pyro-printing to integrate microlenses on the chip (Sect. 16.4.4).

\subsubsection{Removable Inserts Concept}

As previously discussed, the main limitation of $\mu \mathrm{IM}$ technology is the low reconfigurability of the mould once its geometry is defined. In fact, any modification to the mould cavity is very expensive. Our approach is based on a fixed master mould and several exchangeable inserts, which can be easily replaced to produce devices with different functionalities. In this way, the complexity of the mould increases, but 
it allows a significant improvement in its versatility. The combination of different micro-manufacturing technologies has been evaluated in order to produce inserts that can be easily assembled in the mould and that can provide the microfeatures required by the LoC design.

Femtosecond laser ablation of steel has been used to produce the negative of the microfeatures required on the plastic devices. This technology allows to process reasonably large surfaces with small depth features and at high removal rate. In addition, it provides geometrical precision and surface roughness values that meet the requirements of the microfluidic application. To implement easy connection of the LoC with external tubing, complex 3D micro features with high aspect ratio have been produced with micro Electro Discharge machining ( $\mu$ EDM) technology.

In the following subsections the design of the LoC, the mould design and the tool insert manufacturing are described in detail.

\subsubsection{Device Design}

The targeted device is an optical stretcher, consisting of two optical fibres, facing each another on the opposite sides of a microfluidic channel. The counter-propagating light beams delivered by the two fibres exert optical forces on the passing cells, trapping and deforming them. A microscopic imaging of the cell deformation for calibrated optical forces allows retrieving the mechanical compliance of each cell. The conceptual design of the device (Fig. 16.1) is made by two shells $(19.5 \mathrm{~mm}$ length, $7.5 \mathrm{~mm}$ large and $0.7 \mathrm{~mm}$ thick) assembled together by femtosecond laser welding [28]. On the lower shell, a microchannel of $100 \mu \mathrm{m}$ width and $90 \mu \mathrm{m}$ depth with final reservoirs of $1 \mathrm{~mm}$ diameter is produced. $\mathrm{V}$-grooves have been realized for the optical fibre housing with depth and width of $180 \mu \mathrm{m}$ and $235 \mu \mathrm{m}$, respectively. Connectors on the upper shell have been conceived to reduce the number of external components involved in the assembly, thus enabling a direct connection of external capillaries to the chip.

\subsubsection{Mould Design}

The mould consists of two cavities, corresponding to the two shells depicted in Fig. 16.1, implemented on two removable inserts (Fig. 16.2b, c).

A filling and packing phase simulation with Autodesk Moldflow Insight $2016^{\circledR}$ has been preliminary performed to verify the device geometry and to define the gate design. It is important to avoid incomplete filling weld lines and voids (Fig. 16.3a) and to validate the set of the identified parameters through the analysis of the shrinkage distribution (Fig. 16.3b), which is expected to be uniform along the whole component.

The versatility of the mould design is ensued by the possibility of modifying each micro feature independently from the others simply replacing the related insert with a new one. Furthermore, the cavities are independent from the feeding system and can 


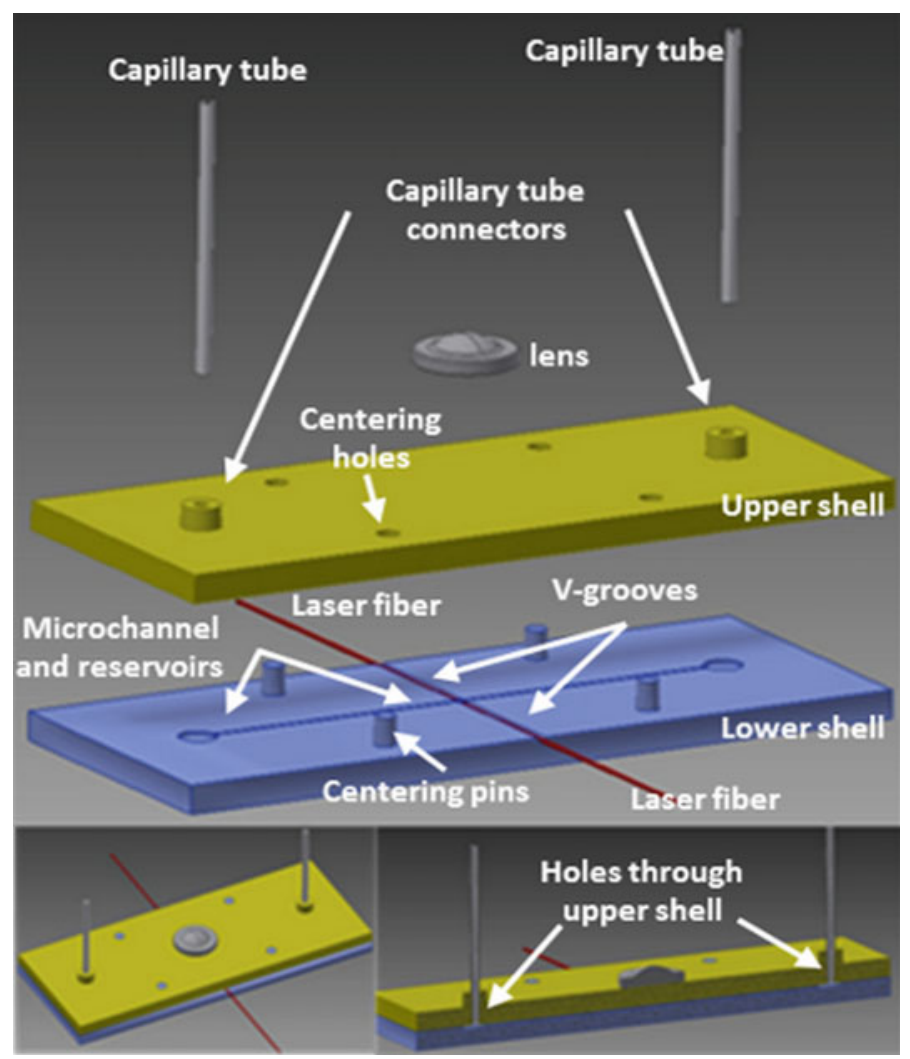

Fig. 16.1 Final design of the optical stretcher LoC developed in this work

be easily replaced thanks to the single sprue that is divided into a double symmetric gate facing the inserts with cavities (Fig. 16.2a).

Since the volume of polymer required for each cavity (about $125 \mathrm{~mm}^{3}$ ) is close to the limit capacity of the micro injection moulding machine $\left(150 \mathrm{~mm}^{3}\right)$, a couple of interchangeable pins having different height are used alternatively to close a runner while the other one is open (Fig. 16.2a-inset) so that one cavity at a time is filled.

\subsubsection{Removable Inserts Fabrication}

A synergic combination of different technological approaches has been adopted for the fabrication of the removable inserts. Traditional milling has been used to machine the tool with its plates and ejection systems, while the bulk cavity has been realized by die-sinking EDM. The inserts most critical features like microchannel, reservoirs and $\mathrm{V}$-grooves have been machined by femtosecond laser micromilling. 


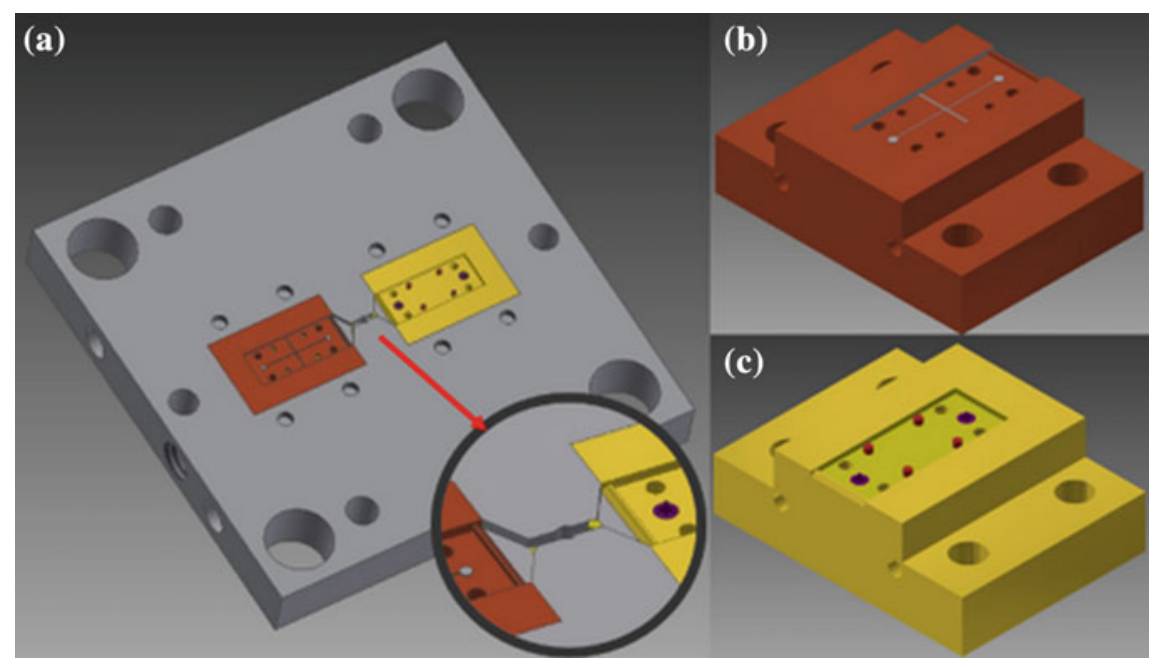

Fig. 16.2 Design of the mould plate with runners and gates (a). Detailed view of the interchangeable pins used to switch between cavities ((a) - inset) and of the cavities inserts $(\mathbf{b}, \mathbf{c})$

(a)

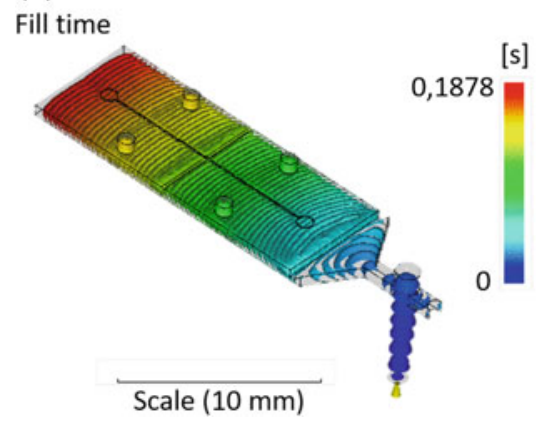

(b)

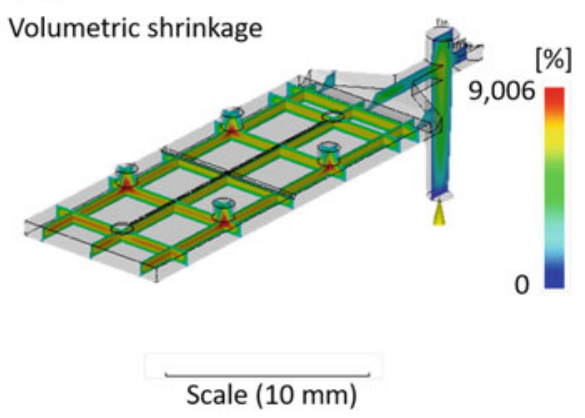

Fig. 16.3 a Polymer filling distribution (time contour plot) and b volumetric shrinkage (section probe plot)

In order to simplify the connection of capillary tubes to the LoC, moulds for access holes were created on the insert corresponding to the top PMMA slide by micro Electro Discharge Machining ( $\mu$ EDM) technology. In Fig. 16.4a the design geometry and dimensions of the tube housing insert are reported. Starting from a cylindrical workpiece, assembled and referred to the main mould, the central conical pin is obtained by eroding the cavities following a layer-by-layer strategy of $0.004 \mathrm{~mm}$ thickness to obtain a smooth surface and avoid step-like effects. Then, a smaller electrode tool, having a diameter of $0.15 \mathrm{~mm}$, has been used to machine the narrow cavity floor at the pin root. 

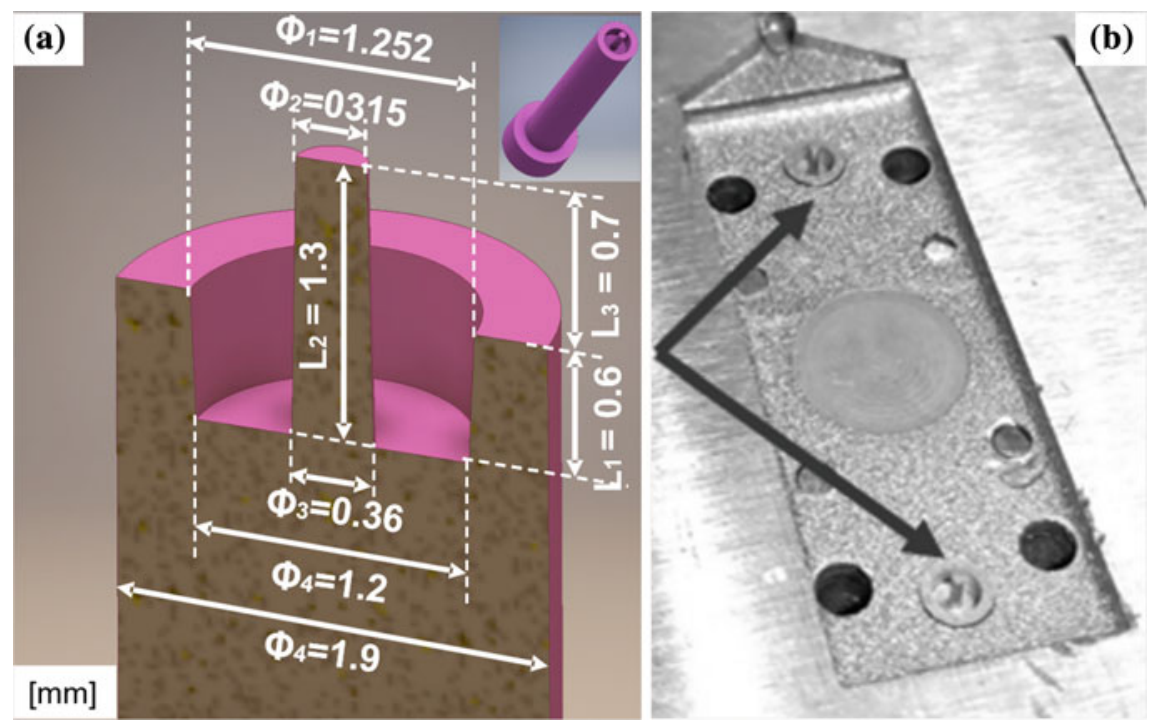

Fig. 16.4 Design of the capillary tube housing insert (a) and picture of its implementation in the $\mu$ EDM-fabricated insert (b)

The same process has been adopted for realizing a smooth circle area of $3 \mathrm{~mm}$ diameter in the centre of the insert (Fig. 16.4b) in order to reduce roughness where the lens, required for the imaging of the samples flowing in the microfluidic channel, will be located.

Femtosecond laser micromachining was used to mill the metal insert containing the complex 3D microfeatures of the bottom layer of the LoC.

Preliminary fs-laser milling tests on steel have been carried out to find the best combination of process parameters, namely the average power, the translation speed and the spacing of the hatch $d$, to get the targeted surface roughness. Each test has been executed on a $3 \mathrm{~mm}^{2}$ area, moving the beam along vertical and horizontal parallel lines. After laser milling, the specimens were ultrasonically cleaned with isopropanol for $5 \mathrm{~min}$ to remove the recast debris. Then, depth and roughness measurements were performed with a confocal microscope. The calculation of the roughness is based on the DIN EN ISO 4287 standard.

The pulse repetition rate has been set at the lowest value delivered by the ultrafast laser source, i.e. $50 \mathrm{kHz}$. In spite of the reduced material removal rate, working at such a low repetition rate produces smoother surfaces because of the reduced thermal load delivered onto the irradiated area [31]. The laser beam was focused and scanned on the insert surface by a galvo-scan head, equipped with an F-theta lens of $100 \mathrm{~mm}$ focal length, so that the beam spot size on the metal surface is about $25 \mu \mathrm{m}$.

Firstly, the beam translation speed was fixed and the influence of the laser average power on the surface quality was investigated. The surface roughness dramatically increases with the laser fluence. For laser power values below $600 \mathrm{~mW}$ a gentle 


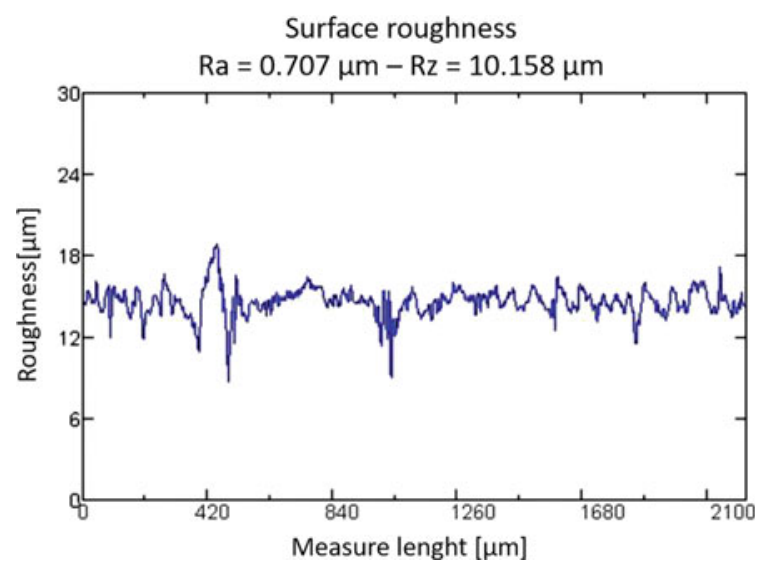

Fig. 16.5 Surface roughness measurement obtained through confocal microscopy (magnification $100 \mathrm{x})$ of the milled surface (200 scanning loops; rep. rate: $50 \mathrm{kHz}$; average power: $0.4 \mathrm{~W}$; hatch: $2 \mu \mathrm{m})$

ablation regime is established producing smooth surfaces. As soon as the laser fluence is increased, ablation occurs through a process referred to as phase explosion, characterized by homogeneous nucleation of bubbles in vapour phase leaving significant pores on the irradiated area and a surface with orange peel morphology and a higher roughness [31].

The influence of the scan speed from $50 \mathrm{~mm} / \mathrm{s}$ to $1 \mathrm{~m} / \mathrm{s}$ on the surface quality has been also investigated. It was noticed that the slower the speed, the less homogeneous is the ablated surface. Furthermore, for the slowest investigated speed values (50 and $100 \mathrm{~mm} / \mathrm{s}$ ) some grooves and pores appear on the surfaces. Therefore, the best results in terms of surface quality are obtained for scan speeds higher than $300 \mathrm{~mm} / \mathrm{s}$, as already confirmed by previous works $[31,32]$. Finally, the inter-line spacing $d$ of the hatch has been optimized. Values of $d$ comprised between 2 and $20 \mu \mathrm{m}$ have been investigated, finding that the most homogeneous and smooth surfaces are obtained for $d=2 \mu \mathrm{m}$.

It has been furthermore verified that the ablation depth of the micromilled area can be finely controlled with micrometre precision by adjusting the number of scanning loops while keeping the surface quality almost unchanged.

Based on the results of the preliminary fs-laser micro-milling tests, the following set of laser parameters has been selected for the fabrication of the mould insert: $50 \mathrm{kHz}$ for the pulse repetition rate, $400 \mathrm{~mW}$ of laser average power, $600 \mathrm{~mm} / \mathrm{s}$ of scanning speed and $2 \mu \mathrm{m}$ of hatch distance. Results of the measurement of surface roughness Ra of a fs-laser milled area of about $600 \mu \mathrm{m}$ length are shown in Fig. 16.5. Ra values below $1 \mu \mathrm{m}$ can be obtained with this set of laser parameters. 

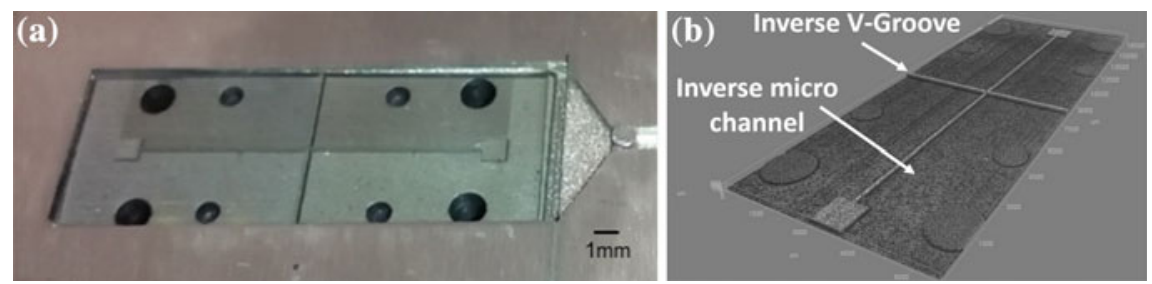

Fig. 16.6 a Picture of the insert machined by fs-laser. b Characterization of the 'negative' microchannel and V-grooves produced on the insert (Zeiss Axio CSM 700)

A picture of the final fs-laser-fabricated mould insert is shown in Fig. 16.6a, whereas Fig. 16.6b shows a confocal image of the same surface. The targeted microfeatures sizes have been successfully reproduced with a tolerance of about $2 \mu \mathrm{m}$ in the worst case.

\subsubsection{Sealing of the Microfluidic Chip}

The two polymeric shells were finally assembled by direct fs-laser welding. Although already successfully demonstrated for glasses, this kind of approach was never applied to transparent polymeric substrates.

Firstly, the combinations of process parameters producing melting of the laser focal volume at low pulse energies and high repetition rates were estimated by solving the heat conduction equations describing the pulsed-laser irradiation of a PMMA substrate [28]. Then, the modifications induced by focusing fs-laser pulses in the volume of the bulk material were experimentally investigated. The goal of this study was to identify an appropriate window of process parameters producing homogeneous and continuous melting, spatially localized in the focal region. This time the laser beam focus was achieved through a 0.3 numerical aperture lens and positioned $1.5 \mathrm{~mm}$ below the surface of a $3 \mathrm{~mm}$-thick PMMA specimen. The beam was then moved along single and multiple parallel lines. Several tests were conducted by operating the laser source at $200 \mathrm{kHz}, 500 \mathrm{kHz}, 1 \mathrm{MHz}, 5 \mathrm{MHz}$ with average power ranging from 0.1 to $1 \mathrm{~W}$. The translation speed was varied from 0.01 to $10 \mathrm{~mm} / \mathrm{s}$. Each test was repeated with $650 \mathrm{fs}$ and 18 ps pulse durations in order to understand the influence of this parameter on the material modification.

The tests demonstrated that the peak power has a significant role in triggering changes of the material. Therefore, we scaled the pulse energies at the two pulse durations to have the same peak power. At both pulse durations, it was evident that heat accumulation is enhanced at higher repetition rates $(>1 \mathrm{MHz})$ and low translation speeds $(<1 \mathrm{~mm} / \mathrm{s})$, in agreement with the simulation results. We observed that better quality results are achieved with 650-fs pulses rather than with 18-ps pulses, therefore we chose the shorter pulses for performing this process. 


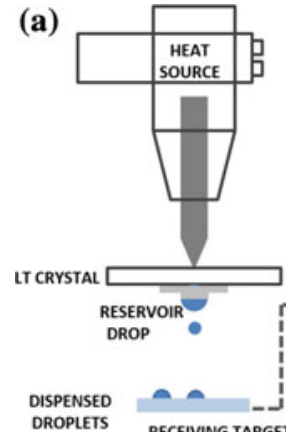

DROPLETS RECEIVING TARGET

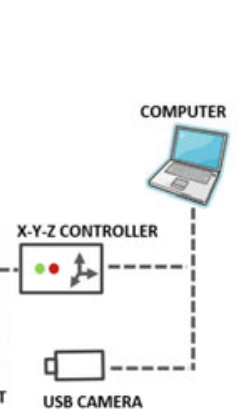

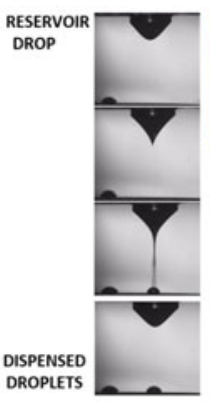

Fig. 16.7 a A schematic set-up of the pyro-EHD printing of polymer microlenses onto microfluidic chip and side view images of a typical sequence of dispensed microlenses. $\mathbf{b}$ Geometrical properties of the deposited lens. $\mathbf{c}$ A pattern of microlenses with different sizes produced on top of a microfluidic device

Based on the results of these preliminary tests, a suitable set of laser parameters was selected producing homogeneous melting in the focal region. The laser source was operated at $5 \mathrm{MHz}$ with pulse energy of $0.4 \mu \mathrm{J}$. The two injection-moulded PMMA shells (Fig. 16.7) composing the optical stretcher were mechanically clamped to achieve an air gap of a few micrometres in the welding area. The laser beam was focused at the interface between the two plates and was moved with a translation speed of $0.1 \mathrm{~mm} / \mathrm{s}$. The laser-welding path surrounded the microfluidic network composed of the two reservoirs, the channel and the two V-grooves.

\subsubsection{On-chip Imaging}

As a preliminary step, the micro-lenses were integrated onto a commercial PMMA microfluidic device (ChipShop $\mathrm{GmbH}$ ) through forward pyro-printing [33]. PyroEHD printing is a novel technique to develop micro-lenses integrated on LoCs while guaranteeing high chemical compatibility, good optical characteristics and flexibility in the design of the realized devices [34].

Micro-lenses fabrication was performed using the printing configuration showed in Fig. 16.7a. A polydimethylsiloxane drop (PDMS Dow Corning Sylgard 184, 10:1 mixing ratio, base to curing agent Midland; $\eta=3900 \mathrm{cP}, \varepsilon p=2.65$ from datasheet), was deposited as a reservoir drop on the pyroelectric crystal and placed above the PMMA device. Once an external heating source is applied onto the crystal, the PDMS drop starts to deform assuming a conical shape, known as Taylor's cone, and dispensing micro-nano and pico-drops [35]. Figure 16.7c shows pictures of a microfluidic device with PDMS micro-lenses pyro-ink-jet-printed on it. Microlenses could present different shapes and dimensions, as a function of the pyro-inkjet 
printing parameters. Once the lenses were deposited on the channel, the device was heated at $80{ }^{\circ} \mathrm{C}$ for $30 \mathrm{~min}$, in order to cure the polymer.

A fluorosilane agent (Fluorolink S10, Solvay Solexis) was used to modify the substrate wettability to avoid excessive spreading of the PDMS lens onto the PMMA substrate. Moreover, the antireflection properties of the coating guarantee good optical properties of the final device. Furthermore, fluorosilane has good chemical resistance and endures abrasion. An $\mathrm{O}_{2}$ plasma treatment (Femto System, Diener Electronic $\mathrm{GmbH} \&$ Co. KG, Ebhausen, Germany) was performed on the microfluidic device to improve the adhesion between the fluorosilane agent and the PMMA substrate.

\subsection{Testing and Validation of Results}

In this section we will present the characterization of the structures fabricated with the techniques and methodologies described in the previous section. We will discuss morphological properties, shape and roughness (Sect. 16.5.1), as well as functional properties, both fluidic (Sect. 16.5.2) and optical (Sect. 16.5.3), and finally we show the operation of the optical stretcher LoC with red blood cells (Sect. 16.5.4).

\subsubsection{PMMA Device Characterization and Strategies to Reduce the Roughness}

The $\mu$ IM production of the two PMMA shells is based on the approach proposed in [36]. The process parameters defined with the simulation and used for the production of the components are reported in Table 16.1 where $T_{\text {melt }}$ is the melt temperature, $T_{\text {mould }}$ is the mould temperature, $V_{i n j}$ is the injection speed, $P_{\text {hold }}$ is the packing pressure, $t_{\text {hold }}$ is the packing time, $t_{\text {cool }}$ is the cooling time and Run is the plunger injection run.

The $\mu$ IM machine used for the device production is a DESMA Tec Formica Plast $1 \mathrm{~K}$ and the polymer Acyrex CM211 PMMA was selected as material for the device fabrication.

The adopted strategy to reduce mould roughness was to use a very low spark energy level during $\mu \mathrm{EDM}$ milling and to adjust the number of scanning loops during femtosecond laser ablation. With the selected parameters (reported in previous sections) we obtained microfluidic channels with a roughness ( $\mathrm{Ra}$ of $0.59 \mu \mathrm{m})$ and a

Table 16.1 Set of process parameters

\begin{tabular}{l|l|l|l|l|l|l|l}
\hline Material & $\mathrm{T}_{\text {melt }}\left({ }^{\circ} \mathrm{C}\right)$ & $\begin{array}{l}\mathrm{T}_{\text {mould }} \\
\left({ }^{\circ} \mathrm{C}\right)\end{array}$ & $\begin{array}{l}\mathrm{V}_{\text {inj }} \\
(\mathrm{mm} / \mathrm{s})\end{array}$ & $\mathrm{P}_{\text {hold }}($ bar $)$ & $\mathrm{t}_{\text {hold }}(\mathrm{s})$ & $\mathrm{t}_{\text {cool }}(\mathrm{s})$ & Run $(\mathrm{mm})$ \\
\hline PMMA & 250 & 80 & 100 & 1000 & 5 & 5 & 10 \\
\hline
\end{tabular}



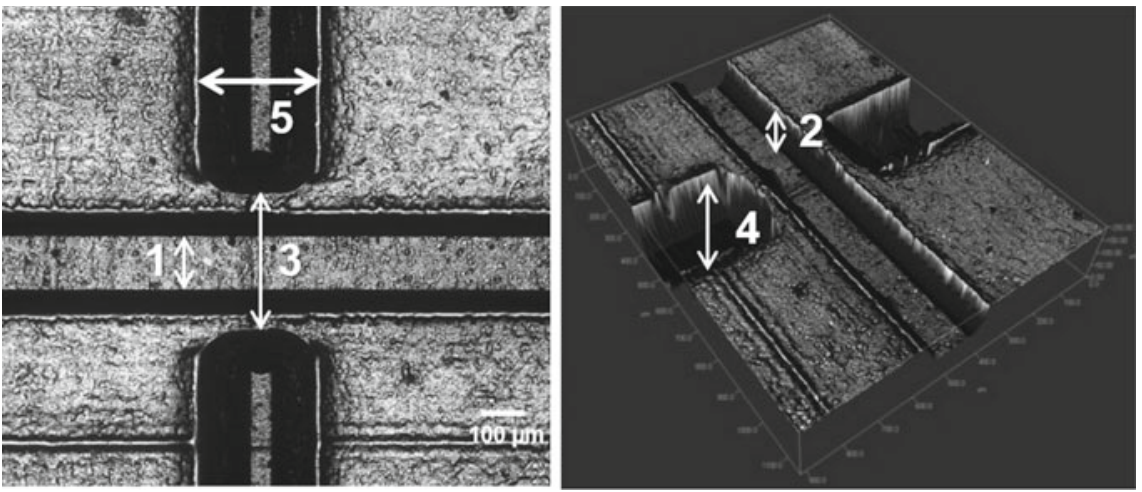

Fig. 16.8 Optical microscope image (left) and confocal microscope image (right) of the microchannels and V-grooves. The critical parameters are: 1: channel width, 2: channel depth, 3: V-groove fibre distance, 4: V-groove depth and 5: V-groove width. The obtained dimensions are within 6\% of the expected ones (measurements done with confocal microscope ZEISS AXIO CSM 700)

shape that match the designed values (see Fig. 16.8). The surface of the access holes on the upper shell presented a relatively low roughness ( $\mathrm{Ra}$ of $0.37 \mu \mathrm{m})$ if compared with raw material. The capillary tube was easily inserted in the access hole providing a good housing adhesion when the fluid flew.

Finally, the V-grooves were realized orthogonal and coaxial to each other (Fig. 16.8) to allocate two standard optical fibres perfectly aligned and emitting two counter-propagating beams at a height of $20 \mu \mathrm{m}$ with respect to the bottom of the microchannel. This height was chosen to maximize the number of cells intercepted by the optical trap. The obtained feature dimensions (see Fig. 16.8) are within $6 \%$ of the expected ones.

To perform optical imaging of the specimens inside the microfluidic channels, it is mandatory to have a channel bottom surface with optical quality. We developed a chemical post-treatment to smoothen the channel surface: the chip was exposed to Chloroform vapour $\left(\right.$ at $40{ }^{\circ} \mathrm{C}$ ) for a few minutes and then annealed at low temperature $\left(80{ }^{\circ} \mathrm{C}\right.$ ) for $20 \mathrm{~min}$ [37]. In this way, the roughness of the surface was reduced (see Fig. 16.9, showing the microchannel bottom before and after the treatment). The improved quality of the surface enabled accurate imaging of the elements flowing in the channel. In addition, this smoothening process is very simple and can be applied in parallel to several chips. Therefore, it relaxes the specifications on the quality of the micromachined sample allowing faster and cost-effective fabrication of the devices. 


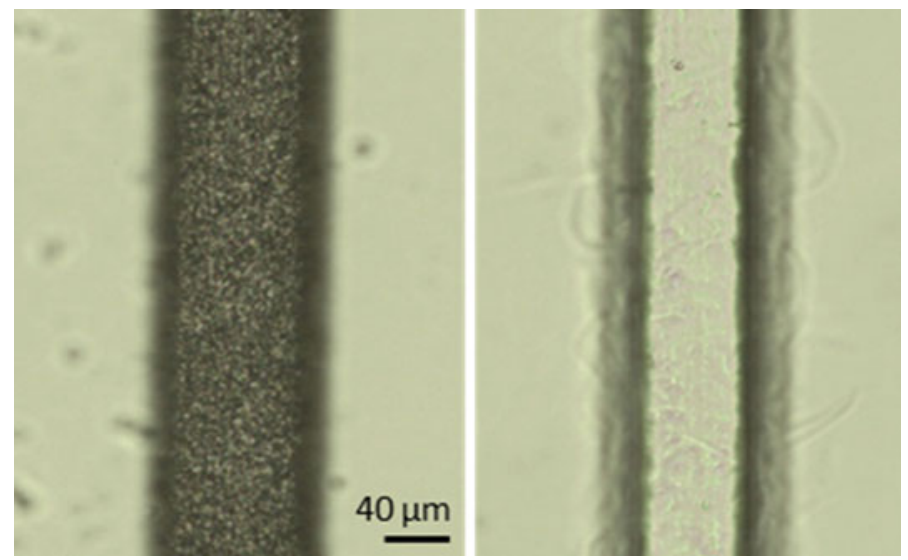

Fig. 16.9 Microscope image (in transmission) of a fs-laser-micromachined channel before (left) and after (right) the chloroform vapour treatment (the roughness is reduced from $\sim 1-2 \mu \mathrm{m}$ to $\sim 300-400 \mathrm{~nm}$ )

\subsubsection{Laser Bonding Characterization}

Firstly, static leakage tests were carried out. A dead-end reservoir, composed of two laser-bonded PMMA slides, one of them preliminarily laser-drilled, was connected to a microfluidic pump (MFCS-EZ, Fluigent) [28]. A blue-coloured liquid was injected at a pressure between 100 mbar and 1 bar with steps of 100 mbar, retaining each value for $3 \mathrm{~min}$. The possible occurrence of fluid leakages was monitored through an optical microscope.

Further leakage tests were executed on a laser milled microchannel, which is the main core of any LoC. The microchannel was fabricated on the surface of a 1-mm-thick PMMA layer by direct femtosecond laser ablation, resulting in a square cross section of $80 \mu \mathrm{m}$ side and a length of $1 \mathrm{~mm}$. The channel connected two laser micro-machined reservoirs, each of them provided with a fs-laser drilled hole. This micro-machined layer was laser bonded to a standard PMMA slide all along the perimeter of the channel and of the reservoirs. A blue liquid was pumped in one reservoir, while the other end was hermetically closed. The leakage test was repeated several times on the same device to demonstrate that the laser bonded contour was able to withstand pressure over time [28].

\subsubsection{Integrated Lenses Characterization}

The lenses were characterized interferometrically. In fact, an interferometric characterization based on Digital Holography (DH) microscopy was carried out to evaluate the optical aberrations of the microlenses and their focusing properties. The optical 
set-up was a classical Mach-Zehnder interferometer in transmission configuration [33]. Once the hologram was recorded, numerical propagation provided the object complex field in whatever plane along the optical axis $z$, from which the intensity and the phase distributions of the optical wave field transmitted by the sample could be extracted. A $2 \mathrm{D}$ fitting was applied to the recovered phase map by using a Zernike polynomial expansion to model and study the optical aberrations produced by the lens. The analysis of the first 10 orders of the linear combination of Zernike polynomials showed that, after the constant offset $(\mathrm{P}=1)$, the main contribution to the development of the Zernike function was a defocus term $(P=5)$. This result was expected because of the spherical shape of the lens. Furthermore, a tilt along the $y$-axis $(\mathrm{P}=3)$ was identified together with a coma aberration along the $\mathrm{x}$-axis $(\mathrm{P}=8)$. The other terms gave a negligible contribution [33]. After completing the optical characterization of the polymeric micro-lenses, their imaging capabilities were tested to show one of their possible applications on a LoC platform. Fibroblast cells were inserted to flow inside the microfluidic channel where the lenses were deposited and aligned. The images of the cells created by the on-chip lens were further imaged by an optical microscope [33]. Figure 16.10a, b show the images of the cells inside and outside the lens area. In particular, these are clearly in-focus only when they flow below the lens, while these are out-of-focus in all the other portions of the channel. In Fig. 16.10a it is also possible to notice the magnifying action operated by the lens, as the channel itself appears to be enlarged. Moreover, in order to proof the lens magnification, a test resolution target placed behind the chip is imaged. It is evident that the smallest target segments in the red box of Fig. 16.10c are not visible at all, while these get resolved when observed through the lens (see the area in the red box in Fig. 16.10d) [33].

\subsubsection{Validation of the LoC Device: Stretching of Red Blood Cells}

The integrated optical stretcher (Fig. 16.1) consists of a central microfluidic channel (width $100 \mu \mathrm{m}$, depth $90 \mu \mathrm{m}$ ) which is crossed perpendicularly by the light beams coming from the two fibres. After some preliminary experiments, it was clear that the fibres should arrive directly to the microfluidic channel without any plastic wall in front of them. In fact, the power required to trap and stretch cells is sufficient to deform and damage the thin polymeric material that was initially left in front of the fibre to improve the channel sealing. The removable insert was thus modified with the femtosecond laser in order to remove the small wall between the fibre ducts and the central microfluidic channel.

An image of the device is shown in Fig. 16.11. The cross-shaped trace of the laser bonding is clearly visible. It should be noted that the welding trajectory of the fs-laser has been changed several times depending on the specific chip design, further evidencing the simple reconfigurability of femtosecond laser processing. The 


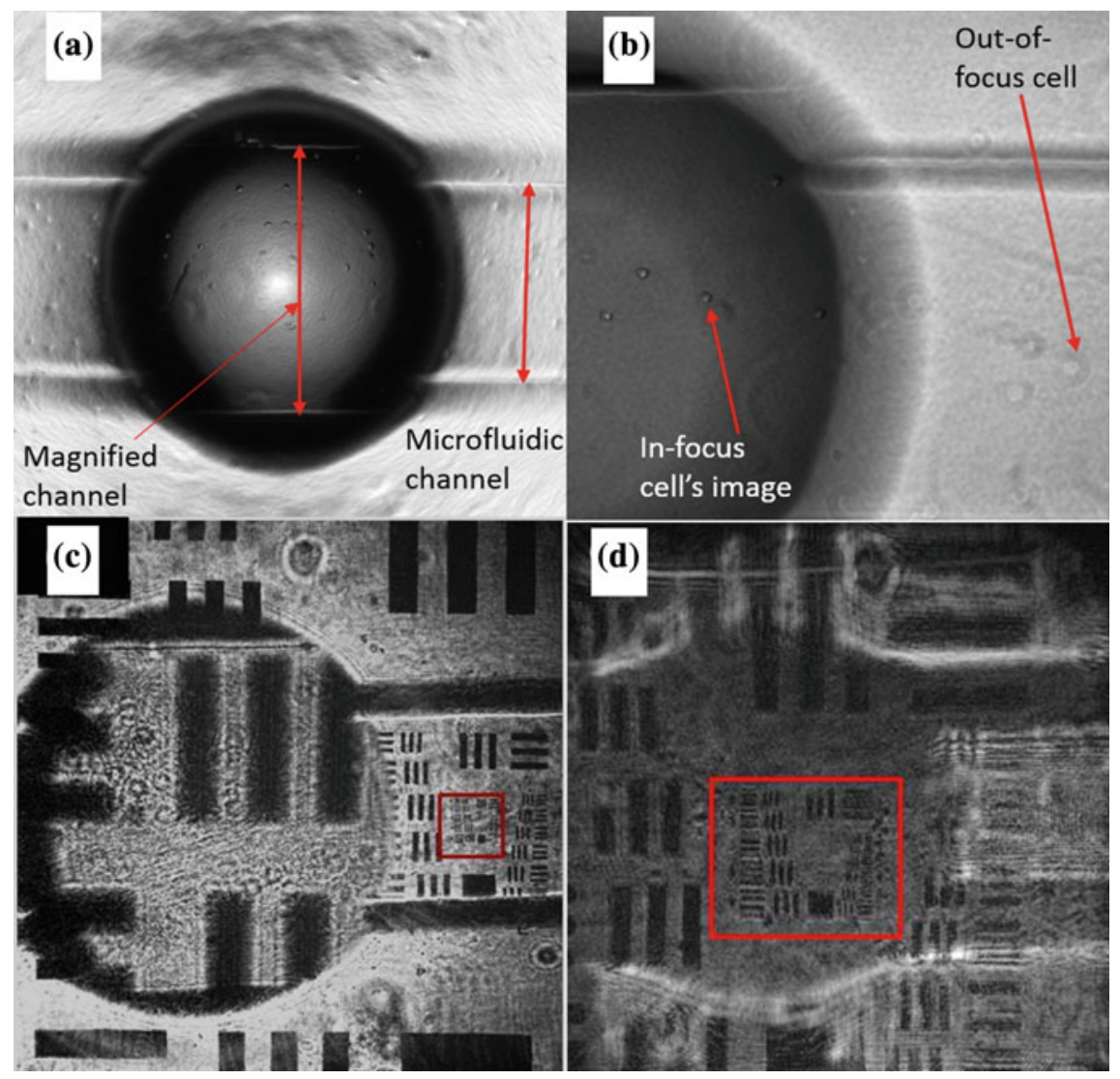

Fig. 16.10 Image of cell flowing inside a microfluidic channel with a plastic lens deposited on the chip surface. $\mathbf{a}$ and $\mathbf{b}$ details of the flowing cells, $\mathbf{c}$ and $\mathbf{d}$ imaging of a test resolution target

fibres were glued in their specific holders. It is important to point out that no further alignment of the fibres is needed as they are forced to follow the ducts that are precisely moulded.

Figure 16.12a shows the experimental set-up that was designed to demonstrate the effectiveness of the integrated optical stretcher. A continuous ytterbium fibre laser (YLD-5 k-1070, IPG Fibertech), characterised by an emitting power up to $5 \mathrm{~W}$ at $1070 \mathrm{~nm}$, was used as light source. The beam coming from the laser was split into two branches by means of a 50-50\% fibre coupler, all the components were single mode at the working wavelength as well as the spliced bare end-fibres (HI-1060-Corning) [27]. The chip was mounted on an inverted microscope, equipped for phase contrast microscopy (Leica), to image the cells in the integrated microfluidic channel. Phase 
Fig. 16.11 Picture of the plastic optical stretcher after the final assembly

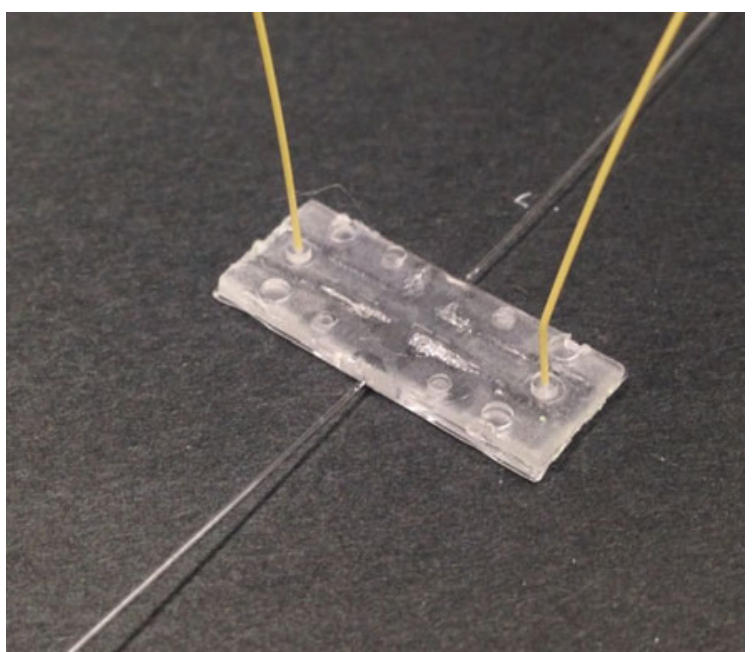

contrast images of optical trapping and stretching of cells were acquired by a CCD camera (DFC310 FX, Leica) through a bandpass optical filter rejecting the infrared light (FGS550, Thorlabs).

We tested the trapping and stretching capabilities of the prototype by using red blood cells. We prepared a solution of $10 \mu \mathrm{L}$ of blood in $8 \mathrm{~mL}$ of hypotonic solution, in which the Red Blood Cells (RBC) acquired a quasi-spherical shape with a radius of $\sim 4 \mu \mathrm{m}$. The cell suspension was transported in the microfluidic channel by an external microfluidic pump (MFCS-EZ, Fluigent). The value of the cell speed was set in the 10-50 $\mu \mathrm{m} / \mathrm{s}$ range for an easy imaging of the flowing cells. RBCs optical trapping was achieved with an estimated optical power at each fibre output of about 30 $\mathrm{mW}$. When a single cell was stably trapped in the microchannel it could be stretched along the trap axis by simultaneously increasing the optical forces applied to the cell by the two counter-propagating beams [27]. Figure 16.12 shows the image of a trapped RBC inside our chip (b), an increase of the optical power in the two fibres clearly produced the stretching of the cell (c). The current device suffered from the limitation of the surface roughness (measured to be $\sim 600 \mathrm{~nm}$ ), which did not allow to achieve optimal quality images. This limit could be overcome by making the chloroform vapour treatment to the channel before welding it to the top slab, as explained in Sect. 16.5.3. Figure 16.12d shows the image of a stretched RBC inside a microfluidic channel after the chloroform treatment, indeed the imaging performance was extremely improved. With this image quality, it will be possible to digitally analyse the deformability of the trapped cells. 

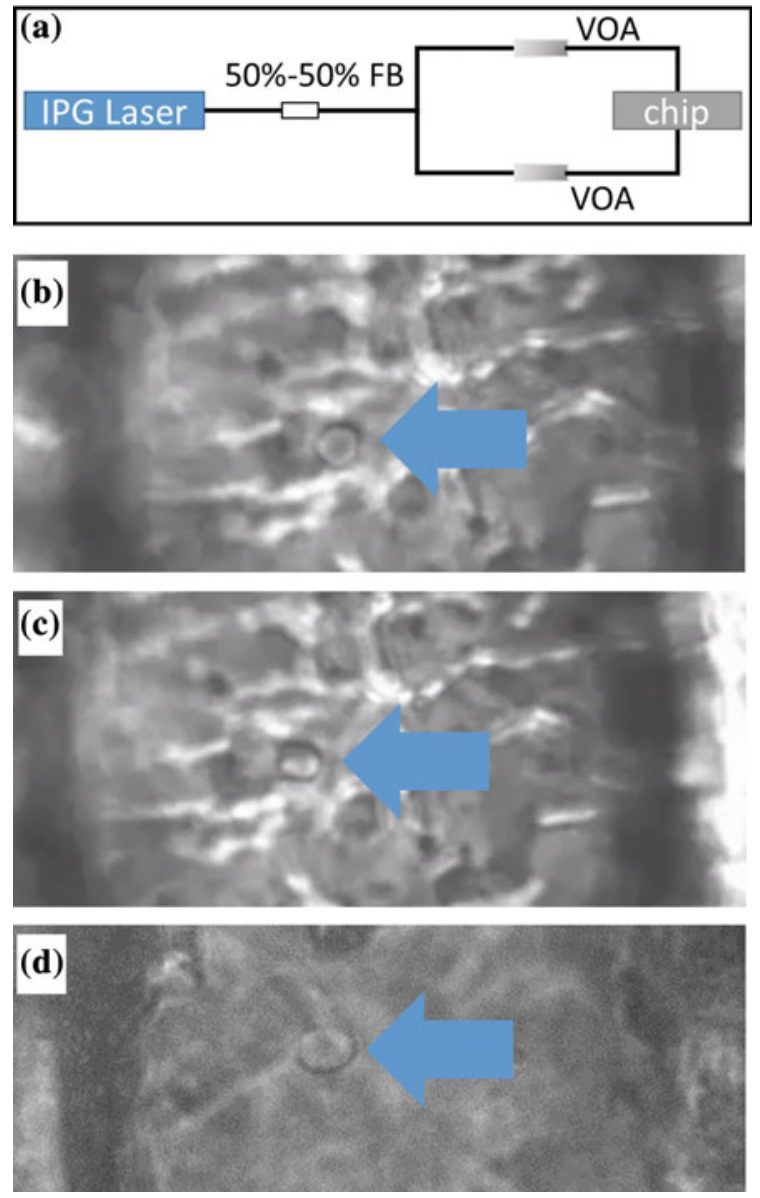

Fig. 16.12 a Scheme of the experimental setup used to demonstrate the LOC working principle. b Microscope image of a trapped red blood cell c microscope image of a stretched red blood cell d image of the stretched red blood cell inside a chloroform treated channel (radius of a RBC 4 $\mu \mathrm{m}$ )

\subsection{Conclusions and Future Perspectives}

The main goals of work have been successfully achieved. The concept of a mould tool with removable inserts has been fully demonstrated. The steel inserts have been ablated by a femtosecond laser according to the design of the integrated optical stretcher and to accurate numerical simulations of the micro injection moulding process.

The processing parameters for the laser ablation of the inserts have been carefully optimized in order to achieve the best surface roughness and the highest fidelity to the design. 
We have designed and demonstrated a new access-hole concept for easy plugging external capillaries to the chip. These access-holes were directly moulded in the top shell of the LoC and make the device extremely compact and easy to connect to the external microfluidic circuit.

In addition to the design flexibility allowed by femtosecond laser micromachining, we have also taken advantage of micro-injection moulding by producing hundreds of devices in a short time. In particular, we have created two different generations of inserts for the top and bottom halves of the integrated optical stretcher during the research activity. This would not have been possible, in terms of both time and cost, if it were not for the new manufacturing approach introduced here.

We have also demonstrated the possibility to weld the two slides together with a femtosecond laser, without the need of any intermediate adhesive layer. This confirms the femtosecond laser as a very versatile tool, capable of solving several important tasks that perfectly complement the injection moulding of lab-on-chip devices.

For the integrated imaging of the microchannel content, we have developed a method to fabricate polymer microlenses directly on top of microfluidic chips. This method, based on a pyro- electrohydrodynamic ink-jet technique, allows one to deposit droplets of PDMS on top of the microfluidic chip that will act as optical lenses. Through the translation of the chip, using a computer-controlled XY stage, an array pattern of polymer microlenses with various shapes was formed.

We can resume the main technological outputs of this work in three points:

- Adding rapid-prototyping capabilities to injection moulding, using the removable insert approach together with femtosecond laser machining of the inserts.

- Demonstrating that optical integration on-chip is possible, fabricating plastic lenses directly on the device surface.

- Increase the portability and reduce the cost of the biosensing systems, by the demonstration of an optical cell manipulator with different functionalities integrated on it.

In terms of perspectives, the developed approach was further exploited in the demonstration of a new prototype of a fully portable plastic LoC for flow cytometry coupled to a cell-phone [38]. This second application required a completely different LoC layout, which was developed and optimized in a very short time exploiting femtosecond laser micromachining of the removable inserts. The versatility of $\mu$ IM enhanced by fs-laser micromachining has the potential of greatly reducing the development time and cost of new LoC devices. These advantages will be particularly beneficial for small-medium enterprises that are very dynamic in developing new products, but do not have the economic capability to produce many different moulds for the optimization of the LoC layout. In addition, this manufacturing versatility will favour custom-oriented solutions that are particularly relevant in the LoC market where there is not yet a single application requiring large volume productions, but more diversified applications requiring different small productions. 
Acknowledgements This work has been funded by the Italian Ministry of Education, Universities and Research (MIUR) under the Flagship Project "Factories of the Future - Italy" (Progetto Bandiera "La Fabbrica del Futuro") [39], Sottoprogetto 1, research projects "Plastic Lab-on-a-chip fabricated by Ultrafast laser Sources" (PLUS) and "Imaging Citometry in Plastic Ultra-mobile Systems" (IC+).

\section{References}

1. Whitesides GM (2006) The origins and the future of microfluidics. Nature 442:368-373

2. Abgrall P, Gue AM (2007) Lab-on-chip technologies: making a microfluidic network and coupling it into a complete microsystem-a review. J Micromech Microeng 17:R15-R49

3. Figeys D, Pinto D (2000) Lab-on-a-chip: a revolution in biological and medical sciences. Anal Chem 72:330-335

4. Kangning Ren K, Zhou J, Wu H (2013) Materials for microfluidic chip fabrication. Acc Chem Res 46:2396-2406

5. Malinauskas M, Žukauskas A, Hasegawa S et al (2016) Ultrafast laser processing of materials: from science to industry. Light Sci Appl 5:1-14

6. Osellame R, Hoekstra HJ, Cerullo G et al (2011) Femtosecond laser microstructuring: an enabling tool for optofluidic lab-on-chips. Laser Photon Rev 5:442-463

7. Sugioka K, Xu J, Wu D et al (2014) Femtosecond laser 3D micromachining: a powerful tool for the fabrication of microfluidic, optofluidic, and electrofluidic devices based on glass. Lab Chip 14:3447-3458

8. Mogensen KB, Klank H, Kutter JP (2004) Recent developments in detection for microfluidic systems. Electrophoresis 25:3498-3512

9. Charrière F, Marian A, Montfort F et al (2006) Cell refractive index tomography by digital holographic microscopy. Opt Lett 31:178-180

10. Giboz J, Copponnex T, Méle P (2007) Microinjection molding of thermoplastic polymers: a review. J Micromech Microeng 17:R96-R109

11. Attia UM, Marson S, Alcock JR (2009) Micro-injection moulding of polymer microfluidic devices. Microfluid Nanofluid 7:1-28

12. Guber AE, Heckele M, Hermann et al (2004) Microfluidic lab-on-a-chip systems based on polymers-fabrication and application. Chem Eng J 101:447-453

13. Becker H, Locascio LE (2002) Polymer microfluidic devices. Talanta 56:267-287

14. Sun Y, Kwok YC (2006) Polymeric microfluidic system for DNA analysis. Anal Chim Acta 556:80-96

15. Chichkov BN, Momma C, Nolte S et al (1996) Femtosecond, picosecond and nanosecond laser ablation of solids. Appl Phys A 3:109-115

16. Schille J, Schneider L, Loeschner et al (2011) Micro processing of metals using a high repetition rate femtosecond laser: from laser process parameter study to machining examples. In: Proceedings of ICALEO: 30th international congress on applications of lasers \& electro-optics, Orlando, FL

17. Neuenschwander B, Bucher GF, Hennig G et al (2010) Processing of dielectric materials and metals with ps laser pulses. In: Proceedings of ICALEO: the 29th international congress on applications of lasers \& electro-optics, Anaheim, California

18. Eaton SM, De Marco C, Martinez Vazquez R et al (2012) Femtosecond laser microstructuring for polymeric lab-on-chips. J Biophotonics 5:687-702

19. Richter S, Döring S, Tünnermann A et al (2011) Bonding of glass with femtosecond laser pulses at high repetition rates. Appl Phys 103:257-261

20. Watanabe W, Onda S, Tamaki T et al (2007) Joining of transparent materials by femtosecond laser pulses. In: Proceedings of SPIE 6460, commercial and biomedical applications of ultrafast lasers VII, 646017, 13 Mar 2007 
21. Tamaki T, Watanabe W, Nishii J et al (2005) Welding of transparent materials using femtosecond laser pulses. Jpn J Appl Phys 44:687-689

22. Watanabe W, Tamaki T, Ozeki Y et al (2010) Filamentation in ultrafast laser material processing. In: Yamanouchi K, Gerber G, Bandrauk, AD (eds) Progress in ultrafast intense laser science VI. Springer Series in Chemical Physics, vol 99. Springer, Heidelberg, pp 161-181

23. Tamaki T, Watanabe W, Itoh K (2006) Laser micro-welding of transparent materials by a localized heat accumulation effect using a femtosecond fiber laser at $1558 \mathrm{~nm}$. Opt Express 14:10460-10468

24. Bishara W, Zhu H, Ozcan A (2010) Holographic opto-fluidic microscopy. Opt Express 18:27499-27510

25. Guck J, Schinkinger S, Lincoln B et al (2005) Optical deformability as an inherent cell marker for testing malignant transformation and metastatic competence. Biophys J 88:3689-3698

26. Lincoln B, Schinkinger S, Travis K et al (2007) Reconfigurable microfluidic integration of a dual-beam laser trap with biomedical applications. Biomed Microdevices 9:703-710

27. Bellini N, Vishnubhatla KC, Bragheri F et al (2010) Femtosecond laser fabricated monolithic chip for optical trapping and stretching of single cells. Opt Express 18:4679-4688

28. Volpe A, Di Niso F, Gaudiuso C et al (2015) Welding of PMMA by a femtosecond fiber laser. Opt Express 23:4114-4124

29. Sobieranski AC, Inci F, Tekin HC et al (2015) Portable lensless wide-field microscopy imaging platform based on digital inline holography and multi-frame pixel super-resolution. Light Sci Appl 4:e346

30. Ferraro P, Coppola S, Paturzo M et al (2010) Dispensing nano-pico droplets and liquid patterning by pyroelectrodynamic shooting. Nat Nanotechnol 5:429-435

31. Bartolo P, Vasco J, Silva B et al (2006) Laser micromachining for mould manufacturing: I. The influence of operating parameters. Assem Autom 26:227-234

32. Lee S, Yang D, Nikumb S (2008) Femtosecond laser micromilling of Si wafers. Appl Surf Sci 254:2996-3005

33. Vespini V, Coppola S, Todino M et al (2016) Forward electrohydrodynamic inkjet printing of optical microlenses on microfluidic devices. Lab Chip 16:326-333

34. Coppola S, Vespini V, Nasti G et al (2014) Tethered pyro-electrohydrodynamic spinning for patterning well-ordered structures at micro- and nanoscale. Chem Mater 26:3357-3360

35. Vespini V, Coppola S, Grilli S et al (2011) Pyroelectric adaptive nanodispenser (PYRANA) microrobot for liquid delivery on a target. Lab Chip 11:3148-3152

36. Trotta G, Volpe A, Martínez Vázquez R et al (2016) Design and fabrication of a mould with multiple inserts for a polymeric microfluidic device. In: International conference on multi material micro manufacturing-4M2016. https://doi.org/10.3850/978-981-11-0749-8 733

37. De Marco C, Eaton SM, Martínez Vázquez R et al (2013) Solvent vapor treatment controls surface wettability in PMMA femtosecond-laser-ablated microchannels. Microfluid Nanofluid 14:171-176

38. Martínez Vázquez R, Trotta G, Volpe A et al (2017) Rapid prototyping of plastic lab-ona-chip by femtosecond laser micromachining and removable insert microinjection molding. Micromachines 8:328-336

39. Terkaj W, Tolio T (2019) The Italian flagship project: factories of the future. In: Tolio T, Copani G, Terkaj W (eds) Factories of the future. Springer 
Open Access This book is licensed under the terms of the Creative Commons Attribution 4.0 International License (http://creativecommons.org/licenses/by/4.0/), which permits use, sharing, adaptation, distribution and reproduction in any medium or format, as long as you give appropriate credit to the original author(s) and the source, provide a link to the Creative Commons licence and indicate if changes were made.

The images or other third party material in this book are included in the book's Creative Commons licence, unless indicated otherwise in a credit line to the material. If material is not included in the book's Creative Commons licence and your intended use is not permitted by statutory regulation or exceeds the permitted use, you will need to obtain permission directly from the copyright holder. 